\title{
reviews
}

\section{Exploration of the Solar System}

J. Veverka

The Realm of the Terrestrial Planets. By Z. Kopal. Pp. 223. (The Institute of Physics: Bristol, UK, 1979.) £7.50.

ThIS book is described as a completely up-to-date account of recent advances in the exploration of the Solar System. Written by a well known astronomer who has made important contributions to our understanding of the Moon and the Solar System, the book is well illustrated and pleasant to read. Unfortunately, it abounds in factual errors. From the nature of most of these, it is clear that the author is writing about the Solar System based on information available, for the most part, a decade ago. It is certainly not an up-to-date account of our current understanding of the terrestrial planets, and is especially dangerous to the initiated reader who has no way of distinguishing fact from error.

The factual errors are too numerous to list in detail. For example, in one single chapter, which consists of 39 pages, there are more than a dozen serious factual errors, not to mention an equally large number of potentially misleading and inaccurate statements. For instance, there is much confusion about the polar caps of Mars. Even though recent Viking results are alluded to, no distinction is made between the annual $\mathrm{CO}_{2}$ frost caps which form every year and disappear during spring and summer, and the permanent (or residual) polar caps, which survive through the Martian summer. In fact, on page 114 , we find a restatement of the old misconception that the southern polar cap of Mars vanishes completely on occasion during summer. It is now known that, although the annual cap is composed of carbon dioxide, the residual caps consist of vast deposits of frozen water, a fact apparently unknown to the author. He is unaware of the fact that even the annual caps can have substantial thickness in places because we read on page 119 that the snowfields in the Martian polar regions must be "less than a few centimetres deep", although numerous studies in recent years have shown that several metres of frost can be deposited in the polar regions during Martian winter.

On page 124, we find further evidence of a thorough misunderstanding of Viking data. It is stated the
Martian sky appears pink in daytime, but that it is blue near sunset and sunrise. There is no evidence to support such an assertion, or the claim that the atmosphere is dust free after sunrise but becomes dusty as the sun rises higher and convection begins to lift up surface dust. Published analyses of the Viking Lander data by Pollack and others clearly demonstrate that the dust does not settle out of the atmosphere daily, but remains suspended for periods on the order of the Martian year. There is no evidence in the Viking data to substantiate the statement made on page 125 that a significant number of dust particles are stirred and lifted into the atmosphere by diurnal convection.

On the same page, there is further evidence that the author has not kept abreast of Mars studies in the last seven years. He states that:

"While the bright markings were identified correctly with arid desert plains and their colour ascribed to that of ferric oxides long before the Viking Landers provided the final proof, the dark markings were thought to owe their lower reflectivity to moisture and even to vegetation flourishing in the spring and watered by systems of canals, natural or artificial from the melting polar caps. Their seasonal variations were regarded as indications that at least plant life existed on this planetary neighbour of ours. These expectations were destined to total disappointment by the outcome of the Viking missions."

This is an astounding statement! Soon after the first Mariner 9 pictures were analysed in 1971-72, it became clear that there is no vegetation in the dark areas of Mars, and that seasonal variations are due to the redistribution of dust, by Martian winds. Similarly, at about the same time, Earth-based telescopic measurements by McCord and others clearly demonstrated that the dark areas of Mars are dark because they are less oxidised than the bright areas. No one in the last seven years has proposed seriously that the dark areas are dark either because of moisture, or because of vegetation.

There are several indications that Kopal does not believe or is not convinced that the large structures such as Olympus Mons are really volcanoes. Although one could sympathise with such a cautious attitude, it is much more difficult to take kindly unsupported statements such as that the whole of Olympus Mons is not more than $10 \mathrm{Myr}$ old.

Perhaps the most colossal blunder in the entire chapter involves sinuous channels. Kopal shows a Mariner 9 picture of some faults on Mars which have never been called 'sinuous channels' by anyone, except Kopal, and have have never been attributed to erosions by liquid water by anyone On the following page, he shows an example of similar features on the Moon and writes, "Note the striking similarity between these lunar features and those on Mars as shown in the previous plate. The origin of these features on the Moon has nothing whatever to do with water". Kopal cautions the reader "against jumping to conclusions about erosion caused by flowing water. For whatever may be a merit of such a suggestion as regards to Mars, it certainly would not apply to the Moon". The whole argument is embarrassing. Nowhere does Kopal show a real Martian sinuous channel. Of course, linear faults were not produced by flowing water.

One should not get the impression that the Mars discussion is the only one marred by serious errors. For example, the discussion of the Mars satellites, Phobos and Deimos, covers only five pages, but contains at least four serious errors. The largest crater on Phobos is not $3 \mathrm{~km}$ across; Phobos is not the darkest body in the Solar System : grooves on Phobos most likely do not represent the effects of "cosmic abrasion"; and it is certainly not true that such grooves are "also characteristic of certain parts of the Moon".

There is little point in picking apart other chapters in similar detail-most contain similar serious factual errors and misconceptions.

The Realm of the Terrestrial Planets, although a superficially attractive, well illustrated book, is too full of errors to be recommended to any reader, except as a record of what a noted astronomer thinks the inner Solar System might be like, based mostly on data generally available about ten years ago. It could have been a much more important book if it were really based on current information.

Joseph Veverka is Associate Professor of Astronomy at Cornell University, Ithaca, New York. 